\title{
Editorial: The World Is Nonlocal
}

\author{
Stewart A. Silling ${ }^{1} \cdot$ Erdogan Madenci $^{2}$
}

Published online: 15 April 2019

(C) Springer Nature Switzerland AG 2019

Nonlocal modeling has come a long way. Researchers in the continuum mechanics and computational mechanics communities increasingly recognize that nonlocality is essential in realistic mathematical models of many aspects of the physical world.

Physical interaction over a finite distance is fundamental at the atomic and nanoscale level, in which atoms and molecules interact through multibody potentials. Long-range forces partially determine the mechanics of surfaces and the behavior of dissolved molecules and suspended particles in a fluid. Nonlocality is therefore an important feature of any continuum model that represents these physical systems at small length scales.

Nonlocality can be just as important even where there is no obvious physical mechanism for direct long-range interactions between material points. It has long been known that nonlocal terms arise naturally in deriving homogenized models of heterogeneous media. In a composite material, for example, the constituent materials provide alternative pathways for the transport of heat or momentum. In a comprehensive homogenized material model for composites, the differences in properties between the constituent materials, as well as their microscopic length scales, create pathways and dictate the nonlocal length scale.

Nonlocal diffusion can be an appropriate continuum model of mass transport where large populations of particles undergo random walk processes or Brownian motion. Fractional calculus, which bears a close connection with strongly nonlocal physical models, is a fascinating branch of mathematics whose applications are starting to be explored in greater depth.

Peridynamics is a nonlocal theory of continuum mechanics that permits cracks and other discontinuities to be modeled directly within its basic field equations. Peridynamic material models contain a fundamental length scale that acts as a cutoff distance for nonlocal interactions between nearby particles.

Stewart A. Silling

sasilli@sandia.gov

1 Sandia National Laboratories, Albuquerque, NM, USA

2 University of Arizona, Tucson, AZ, USA
The peridynamic description, which uses integro-differential equations, converges to the traditional PDE theory where there are no discontinuities; in this sense, peridynamics provides a generalization of the PDE theory that enables the direct modeling of cracks. Peridynamic differential operators provide a new way to accurately approximate local equations using the formalism of peridynamic integrals.

Nonlocal constitutive models typically operate on larger numbers of degrees of freedom than in the PDE theory. For example, in peridynamics, the constitutive model maps all the displacements in the neighborhood of a point into bond forces. In a continuous medium, these degrees of freedom are infinite in number, in contrast to the nine independent components of the deformation gradient tensor. The availability of these extra degrees of freedom allows many phenomena to be modeled within a nonlocal theory just by formulating an appropriate constitutive model. For example, wave dispersion curves in peridynamics can be tailored to a particular material by prescribing the dependence of the bond interaction forces on the length of the bond.

Nonlocal modeling therefore provides a rich mathematical framework in which scientists and mathematicians can dream up new ways of reproducing observed phenomena in the physical and biological world, technologies like image processing, transportation, and even social sciences.

The current growth of interest in nonlocal modeling is partially fueled by advances in computer power and numerical algorithms. Today, we have a much greater ability to solve problems with nonlocal models than in the 1960s and 1970s, when some of the pioneering research on nonlocal continuum mechanics was performed. Now, we can write down a nonlocal mathematical model and, with the help of a computer, see what it predicts and how it behaves. The development of faster and more accurate algorithms for solving nonlocal mathematical problems is an active and vital part of nonlocal modeling. This is particularly important because it is often much more difficult to derive analytic solutions in nonlocal theories than in local theories.

The Journal of Peridynamics and Nonlocal Modeling provides a vehicle for researchers to publish the full spectrum of work including fundamental mathematical analysis, physical 
models, mechanical theory, numerical methods, and applications. We hope to encourage new ideas and promote high quality by providing meaningful and constructive reviews. We aim to help achieve mutual awareness and understanding among the practitioners of the many techniques and methods using various aspects of nonlocality. In particular, we hope that researchers in different parts of the world will use the journal as a means to present their work to the international nonlocal modeling community.

The papers in our inaugural issue illustrate the breadth of the journal's scope. Liu and Li present a nonlocal model for material failure by void growth and the model's implementation in ABAQUS. A review article by Diehl provides a much- needed summary of the literature on validation of peridynamics. Celik, Oterkus, and Guven describe an innovative method for deriving the properties of nanoscale films from atomic force microscope measurements using nonlocal modeling. The paper by Prakash presents a novel method for determining peridynamic bond stiffness for materials. Wildman describes a method to reduce excessive wave dispersion in peridynamic simulations.

We gratefully acknowledge the contribution of SpringerNature editors Ms. Melissa Fearon and Mr. HoYing Fan, and the SpringerNature staff, in starting the journal and helping it to get off the ground. 